\title{
A non-DNN Feature Engineering Approach to Dependency Parsing - FBAML at CoNLL 2017 Shared Task
}

\author{
Xian Qian \\ xianqianefb.com \\ Yang Liu \\ yangli@fb.com \\ Facebook Applied Machine Learning / 1 Facebook Way
}

\begin{abstract}
For this year's multilingual dependency parsing shared task, we developed a pipeline system, which uses a variety of features for each of its components. Unlike the recent popular deep learning approaches that learn low dimensional dense features using non-linear classifier, our system uses structured linear classifiers to learn millions of sparse features. Specifically, we trained a linear classifier for sentence boundary prediction, linear chain conditional random fields (CRFs) for tokenization, part-of-speech tagging and morph analysis. A second order graph based parser learns the tree structure (without relations), and a linear tree CRF then assigns relations to the dependencies in the tree. Our system achieves reasonable performance $-67.87 \%$ official averaged macro F1 score.
\end{abstract}

\section{Introduction}

Our system for the universal dependency parsing shared task in CoNLL 2017 (Zeman et al., 2017) follows a typical pipeline framework.

The system architecture is shown in Figure 1, which consists of the following components : (1) sentence segmentor, which segments raw text into sentences, (2) tokenizer that tokenizes sentences into words, or performs word segmentation for Asian languages, (3) morphologic analyzer generates morphologic features, (4) part-of-speech (POS) tagger generates universal POS tags and language specific POS tags, (5) parser predicts tree structures without relations, (6) a relation predictor assigns relations to the dependencies in the tree.

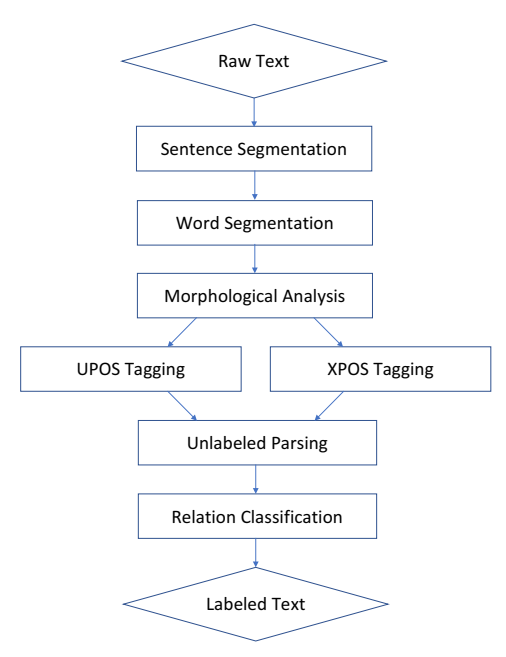

Figure 1: System architecture: pipeline components for universal dependency parsing.

For each component, we take a non deep learning based approach, that is the typical structured linear classifier that learns sparse features, but requires heavy feature engineering.

Sentence segmentation, tokenization, POS tagger and morphologic analyzer are based on linear chain CRFs (Lafferty et al., 2001), and the relation predictor is based on linear tree CRFs. We train the pipeline for each language independently using the training portion of the treebank and the official word embeddings for 45 languages provided by the organizers. Our system components are implemented in C++ with no third party toolkits. Due to the time limit, we did not optimize our system for speed or memory. 


\section{System Components}

\subsection{Sentence Segmentation}

\subsubsection{Task setup}

We cast sentence segmentation as a classification problem at the character level, determining whether a character is the end of the sentence character (EOS). To obtain the gold labels, we aligned the raw text file with the conllu file with annotations.

Since most characters are not sentence boundaries, using all the characters will make the data very imbalanced. To address this problem, we only consider a character as a candidate trigger if it is labeled as EOS at least once in the training data. Intuitively this would prune many characters as EOS characters should be punctuation marks. However, we noticed that for English (possibly other languages too) many sentences in the data end without punctation, and thus the last character of the sentence will be added into the EOS character trigger set. To reduce the size of the triggers, we use a three label scheme for the characters.

- Label $N$ for the character following the end of a sentence, and before the beginning of the next sentence. A typical example for this is the space between two sentences. Even for cases when punctuation marks are omitted, this applies to the space separating the two sentences.

- Label $E$ represents a character is the end of a sentence, and its next character is the beginning of the next sentence. This category is introduced for sentences that are not split by space. For example, past few years,...Great to have you on board!, ' $\mathrm{G}$ ' is the beginning of the second sentence, '? before 'G' has a label of ' $E$ '.

- Label $O$ is used for all other cases. Note that a punctuation mark that ends a sentence will have a label of ' $\mathrm{O}$ ' if there is a space following the sentence. In this case, EOS information is obtained by the ' $\mathrm{N}$ ' label for the space.

Using this scheme, during testing, an EOS character is found if it is labeled as $E$ or its next character is labeled as $N$. For training, we collect the characters labeled as $E$ or $N$ in the training set as candidates. Table 1 shows the number of candidates for each language. This significantly reduces

\begin{tabular}{|c|c|}
\hline languages & \#trigger characters \\
\hline cs_cltt, et, it, lv, pt, pt_br & 3 \\
\hline en_lines, sl & 4 \\
\hline no_nynorsk & 5 \\
\hline no_bokmaal & 6 \\
\hline ru_syntagrus & 7 \\
\hline en & 8 \\
\hline cs & 14 \\
\hline zh & 20 \\
\hline ja & 23 \\
\hline others & 2 \\
\hline
\end{tabular}

Table 1: Number of trigger characters for EOS detection.

the number of trigger candidates compared to considering all the characters.

\subsubsection{Features}

We use a linear classifier for EOS detection. We tune the feature templates on the English development data, and apply to all the other languages. Detailed feature templates are described in Table 2. Features include the surrounding characters and their lower cases. For character types, we use digit and letters, and keep the other symbols. Take 12:00pm as an example, it is represented as: 00:00aa, where we replace all digits by ' 0 ' and all lower cased letters by 'a'. For languages that have spaces between words, we also use the surrounding 'words' split by spaces and the current character. For example, for the following example: comes this story: President Bush for character ' $y$ ', we have word features: wor $_{-2}=$ this, word $_{-1}=$ stor, word $_{1}=$ :, wor $_{2}=$ President .

\subsection{Tokenization}

\subsubsection{Methodology}

We use a sequence labeling model for tokenization. Each character will be labeled as one of the following tags:

- $B$ : beginning of a multi-character token,

- $I$ : inside a multi-character token,

- $E$ : end of a multi-character token,

- $S$ : single character token,

- $O$ : other.

The labels are generated by aligning the raw text with the gold sentence segmentation with the word form column of the conllu table. 


\begin{tabular}{|c|}
\hline $\operatorname{char}_{i},-3 \leq i \leq+3$ \\
\hline char $_{i} \operatorname{char}_{i+1},-3 \leq i \leq+2$ \\
\hline lowchar $_{i},-3 \leq i \leq+3$ \\
\hline lowchar $_{i}$ lowchar $_{i+1},-3 \leq i \leq+2$ \\
\hline chartype $_{i},-3 \leq i \leq+3$ \\
\hline chartype $_{i}$ chartype $_{i+1},-3 \leq i \leq+2$ \\
\hline word $_{i}, i=-2,-1,1,2$ \\
\hline wordtype $_{i}, i=-2,-1,1,2$ \\
\hline
\end{tabular}

Table 2: Feature templates for sentence segmentation. char $_{i}$ is the $i^{\text {th }}$ character to the right of current character, char ${ }_{-i}$ is the $i^{\text {th }}$ character to the left of the current character. lowchar is the lower cased character, chartype is the character type, it can be digit, upper cased letter, lower cased letter or other. word $_{i}$ is surrounding 'words' splitted by spaces and the current character. wordtype is the concatenation of character types

\begin{tabular}{|c|}
\hline chartype $_{0}$, chartype $_{-1}$ chartype $_{0}$ \\
\hline word $_{-1}$, word $_{1}$ \\
\hline word $_{-1}$ chartype $_{0}$, word $_{1}$ chartype $_{0}$ \\
\hline chartype $_{-1}$ chartype $_{0}$, chartype $_{0}$ chartype $_{1}$ \\
\hline transition feature \\
\hline
\end{tabular}

Table 3: Tokenization feature templates for languages with space between words (except Chinese and Japanese).

\subsubsection{Features}

Linear chain CRF is used to learn the model with character and word n-gram features. We used two sets of feature templates, one for languages having spaces between words including English, Arabric etc., the other for languages without spaces including Chinese and Japanese, as shown in Table 3 and 4. The first feature template set is tuned on English development set, the second one is tuned on Chinese development set.

\subsection{POS Tagging and Morph Analysis}

\subsubsection{Methodology}

For morphological analysis and POS tagging, we use the same model setup and features, therefore we group them together in this section. We used linear chain CRFs for these tasks (a sequence labeling task for each word in the sequence). As the morph features consist of several fields separated by a special symbol, we treat the prediction of each field as an independent task, and then combine the predictions from different models. For

\begin{tabular}{|c|}
\hline char $_{i}$ char $_{i+1},-2 \leq i \leq 1$ \\
\hline word $_{-1}$, word $_{1}$ \\
\hline char $_{0}$ word $_{\text {left to current character }}$ \\
\hline char $_{0}$ word $_{\text {right to current character }}$ \\
\hline word $_{\text {left to current character }}$ \\
\hline word $_{\text {right to current character }}$ \\
\hline word $d_{\text {left to left character }}$ \\
\hline word $d_{\text {right to left character }}$ \\
\hline word $_{\text {left to right character }}$ \\
\hline word $_{\text {right to right character }}$ \\
\hline transition feature \\
\hline transition feature + current character \\
\hline
\end{tabular}

Table 4: Tokenization feature templates for Chinese and Japanese. Words in these languages are obtained by maximum forward/backward matching.

POS tagging (both universal (UPOS) and language specific POS (XPOS) tagging), we use the same set of features as used for morph analysis, and the automatically predicted morph features. For languages that have multiple labels in XPOS tag, we use a similar strategy as for morph analysis, i.e., learning multiple taggers and combine the results.

\subsubsection{Features}

The list of feature templates are shown in Table 5. Note for POS tagging, as mentioned above, one additional feature is the morph feature, which comes from the automatic morph models.

The basic features includes word and lower cased word n-grams, prefixes and suffixes. With these features, the baseline UPOS tagger achieves 94.78\% accuracy on the English development set. Since we do not use deep learning based approaches, incorporating pretrained word embeddings is not straightforward for linear classifiers. In our system, we clustered the word vectors using k-means, where $k=2048$ and 10000, and then used the cluster n-grams as features.

\subsection{Unlabeled Dependency Parsing}

\subsubsection{Methodology}

Our dependency parser consists of two components, one is the unlabeled parser which only predicts the tree structures, the other is relation type prediction that assigns dependency relations to the dependencies. Originally, we trained a third order parser with word/POS/morph n-gram features, but it is too slow to extract features, especially 


\begin{tabular}{|c|}
\hline word $_{i},-2 \leq i \leq 2$ \\
\hline word $_{i} \operatorname{char}_{i+1},-2 \leq i \leq 1$ \\
\hline cluster $_{0}$, cluster $_{i}$ cluster $_{i+1}, i=-1,0$ \\
\hline lowerCasedWord $_{0}$ \\
\hline prefi $_{i, j}, i=-1,0,1,1 \leq j \leq 6$ \\
\hline suffix $_{i, j}, i=-1,0,1,1 \leq j \leq 6$ \\
\hline word $_{0}$ prefix $x_{i, j}, i=-1,1,1 \leq j \leq 6$ \\
\hline word $_{0}$ suffix $_{i, j}, i=-1,1,1 \leq j \leq 6$ \\
\hline morph (invalid for morph analysis) \\
\hline transition features \\
\hline
\end{tabular}

Table 5: Feature templates for morph analysis and POS tagging, where prefix $i x_{i, j}$ is the length $=j$ prefix of the $i^{t h}$ word to the right of current word, cluster $_{i}$ is the cluster id of word $_{i}$

the third order features. So we chose to build a second order parser to balance speed and performance. We developed two versions of dependency parsers, one is pseudo-projective parser that handles treebanks that are nearly projective (projective dependencies $\%>95 \%$ ), the other is the 1-endpoint-crossing parser (Pitler et al., 2013; Pitler, 2014) that processes treebanks with more non-projective dependencies (projective dependencies $\%<95 \%$ ), such as Dutch-LassySmall, Ancient_Greek, Ancient_Greek-PROIEL, Basque, Latin-PROIEL and Latin. We modified the original third order 1-endpoint-crossing parsing algorithm to guarantee the unique derivation of any parse tree, because we need the top $\mathrm{k}$ parse trees for training.

\subsubsection{Features}

Our original third order parser includes $1000+$ feature templates, and generated more than 100 million features on English data. As the features consume too much memory, making the parser rather slow, we kept only 260 templates, and use second order parser instead, which generated 15 million features. Most of the feature templates come from the previous works (Koo and Collins, 2010; McDonald et al., 2005), including word, POS ngrams and their combinations. We also add some morphology and word cluster n-grams. Detailed feature templates are described in Table 6.

\subsection{Relation Classification}

\subsubsection{Methodology}

Once the tree structure of a parse tree is obtained, we train a linear tree CRF to assign the relation type to each arc in the tree. Given a tree represented as a collection of arcs: $\mathcal{T}=\{e\}$, the tree CRF represents the potential function of $\mathcal{T}$ as the sum of the potential functions of arcs and arc pair chains:

$$
\phi(\mathcal{T})=\sum_{e} \phi(\mathrm{e})+\sum_{\mathrm{e} \rightarrow \mathrm{e}^{\prime}} \phi\left(\mathrm{e} \rightarrow \mathrm{e}^{\prime}\right)
$$

where $\phi(e)$ is the linear combination of node features in the CRF and $\phi\left(e \rightarrow e^{\prime}\right)$ is the linear combination of transition features in the CRF.

\subsubsection{Features}

For each arc $p \rightarrow c$, we use the same feature templates as in Table 6 to generate node features. For transition features, we simply use the relation type bigrams, i.e., relation $(\mathrm{g} \rightarrow \mathrm{p})$ relation $(\mathrm{p} \rightarrow \mathrm{c})$.

\section{CoNLL Shared Task Results}

\subsection{Implementation details}

All the classifiers, including linear chain CRF, tree CRF and second order dependency parser, are trained using 10-best MIRA (McDonald et al., 2005). Parameters are averaged to avoid overfitting. We found that $k$ best MIRA consistently outperforms averaged perceptron about $0.1-0.2 \%$ for all tasks.

For CRFs and the parser, we used the lazy decoding algorithm (Huang and Chiang, 2005) for fast $k$-best candidate generation, the complexity is nearly the same as 1-best decoding. Specifically, the time complexity for CRFs is $O\left(n L^{2}+\right.$ $n k \log (k))$, and $O\left(n^{4}+n k \log (k)\right)$ for the parser. where $n$ is the length of sentence.

Both CRFs are optimized for fast tagging: strings like words, POS tags are mapped to bit strings for efficient concatenation to generate feature strings, while the parser is not optimized. The actual running time for 1-endpoint-crossing parser is about 1.8 times of projective parser, though theoretically it should be 50x times slower. The main reason is that feature generating is much more slower than decoding, which is actully the same for both parsers. For fast training, we use hogwild strategy to update the parameters using 30 threads. Empirical results on English development data showed that compared with standard MIRA that only used single thread, the hogwild strategy get $5 \mathrm{x}$ speedup, the parser can be trained within 2.5 hours. While the performance is very competitive, only lost $0.1 \%$ UAS. 


\begin{tabular}{|c|}
\hline $\mathrm{p}_{i}$.word,,$\quad \mathrm{c}_{i}$.word,$\quad-2 \leq i \leq 2$ \\
\hline $\mathrm{p}_{i}$.word $\mathrm{p}_{i+1}$.word, \\
\hline $\mathrm{p}_{i}$.word $\mathrm{c}_{j}$.word,,$\quad \mathrm{c}_{i}$.word $\mathrm{s}_{j}$.word,$\quad \mathrm{g}_{i}$.word $\mathrm{c}_{j}$.word $, \quad-1 \leq i, j \leq 1$ \\
\hline $\mathrm{g}_{i}$. word $\mathrm{p}_{j}$.word $\mathrm{c}_{k}$.word,$\quad \mathrm{p}_{i}$.word $\mathrm{c}_{j}$.word $\mathrm{s}_{k}$.word $, \quad-1 \leq i, j, k \leq 1$ \\
\hline $\mathrm{p}_{i}$.word $\mathrm{c}_{j}$.word $\mathrm{c}_{j+1} \cdot$ word $, \quad-1 \leq i \leq 1, \quad-1 \leq j \leq 0$ \\
\hline $\mathrm{p}_{i}$.word $\mathrm{p}_{i+1}$.word $\mathrm{c}_{j}$.word $, \quad-1 \leq i \leq 0, \quad-1 \leq j \leq 1$ \\
\hline $\mathrm{g}_{0} \cdot$ word $\mathrm{p}_{i} \cdot$ word $\mathrm{c}_{j}$.word $\mathrm{c}_{j+1}$.word, \\
\hline $\mathrm{g}_{0}$.word $\mathrm{p}_{i}$.word $\mathrm{p}_{i+1}$.word $\mathrm{c}_{j}$.word \\
\hline $\mathrm{p}_{i}$.word $\mathrm{c}_{j}$.word $\mathrm{c}_{j+1}$.word $\mathrm{s}_{0}$.word, $, \quad-1 \leq i \leq 1, \quad-1 \leq j \leq 0$ \\
\hline $\mathrm{p}_{i}$.word $\mathrm{p}_{i+1}$.word $\mathrm{c}_{j}$.word $\mathrm{s}_{0}$.word, $\quad-1 \leq i \leq 0, \quad-1 \leq j \leq 1$ \\
\hline replace word above by upos, xpos, lowCasedWord, wordCluster, morph \\
\hline combine the templates above with distance and direction of arcs \\
\hline
\end{tabular}

Table 6: Feature templates for unlabeled dependency parsing, where $\mathrm{p}_{i}, \mathrm{c}_{i}, \mathrm{~g}_{i}, \mathrm{~s}_{i}$ are the $i^{\text {th }}$ token right to the parent, child, grand parent, sibling. (to the left, if $i<0$ )

To cluster word vectors, we implemented fast $k$ means using triangle inequality. We let $k$ means run 20 iterations using 45 threads to quickly generate clusters. For languages without pretrained word vectors, such as en_lines, we use word vectors from en instead.

For surprised languages, we trained POS tagger, morphological analyzer and parser using the example data. The word cluster features are derived by running word $2 \mathrm{vec}$ on the unlabeled dataset, and k-means clustering. For sentence segmentation and tokenization, we just used the models trained on English data, since the example dataset is quite limited.

\subsection{Results on development data}

The feature sets are tuned on English development data, except some languages specific tasks such as Chinese word segmentation. Table 7 shows the results on development dataset. We have the following observation regarding feature effect.

- Character type features are useful for sentence segmentation, which made $13 \%$ absolute $\mathrm{F} 1$ score improvement.

- Morphological features help the parser, resulting in an UAS $0.5 \%$ absolute F1 score improvement.

- For tokenization, word features i.e., word $_{-1}$ and word $_{1}$ in Table 3 are useful, which made $1 \%$ absolute $\mathrm{F} 1$ score improvement.

- Lemma features do not have a big effect on parsing. We compared using the gold lemma features vs. the automatically generated ones, with about $0.3 \%$ improvement from the former, and only $0.1 \%$ using the latter. Because of this our system did not do lemmartization for all the languages.

- Word cluster features have limited gains. We tried two different ways to convert the pretrained word vectors to binary features:

(1) find the $k$ nearest neighbors ( $k=3$ in experiments) in the embedding space, and use these neighors as features;

(2) cluster the words into $k$ clusters, $(k=$ $8,16, \ldots, 2048,10000,100000)$, and used the cluster features.

The results on the English development set showed that the two approaches performed quite the same, both achieving $94.92 \%$ UPOS accuracy, $0.15 \%$ improvement over the baseline. In addition, we noticed that the word cluster features did not help when $k$ is small. In our system submission, we used $k=$ 2048,10000 to generate the clusters.

It is worth pointing out that such improvement from using the cluster features is quite limited compared to using embeddings in deep learning based methods. For example, using stacked word and character bi-LSTMCRFs (Lample et al., 2016) achieved $95.75 \%$ POS tagging accuracy, and $96.00 \%$ using word+prefix/suffix embedding. We suspect that the converting real valued features to binary features (cluster features) loses too much information. 


\begin{tabular}{|c|c|c|c|c|c|c|c|}
\hline Language & Sentence & Words & UPOS & XPOS & Feats & UAS & LAS \\
\hline ar & $87.89 \%$ & $92.15 \%$ & $87.51 \%$ & $81.08 \%$ & $78.83 \%$ & $70.33 \%$ & $65.03 \%$ \\
\hline bg & $91.32 \%$ & $99.77 \%$ & $97.36 \%$ & $75.72 \%$ & $89.78 \%$ & $87.75 \%$ & $83.30 \%$ \\
\hline $\mathrm{ca}$ & $99.30 \%$ & $99.73 \%$ & $97.89 \%$ & $97.92 \%$ & $95.63 \%$ & $88.69 \%$ & $85.41 \%$ \\
\hline cs & $96.09 \%$ & $99.96 \%$ & $98.71 \%$ & $92.11 \%$ & $88.65 \%$ & $89.46 \%$ & $86.11 \%$ \\
\hline cs_cac & $99.50 \%$ & $100.00 \%$ & $99.00 \%$ & $88.60 \%$ & $83.94 \%$ & $87.47 \%$ & $84.26 \%$ \\
\hline cs_cltt & $74.36 \%$ & $99.33 \%$ & $89.20 \%$ & $70.17 \%$ & $65.97 \%$ & $71.70 \%$ & $68.37 \%$ \\
\hline da & $87.39 \%$ & $100.00 \%$ & $95.65 \%$ & $0.00 \%$ & $89.51 \%$ & $79.36 \%$ & $75.40 \%$ \\
\hline $\mathrm{de}$ & $93.68 \%$ & $99.91 \%$ & $93.39 \%$ & $95.74 \%$ & $79.83 \%$ & $84.16 \%$ & $79.76 \%$ \\
\hline el & $94.37 \%$ & $99.78 \%$ & $94.76 \%$ & $94.80 \%$ & $84.85 \%$ & $81.99 \%$ & $78.60 \%$ \\
\hline en & $81.43 \%$ & $98.92 \%$ & $93.89 \%$ & $92.22 \%$ & $92.23 \%$ & $81.63 \%$ & $78.11 \%$ \\
\hline en_partut & $95.73 \%$ & $99.37 \%$ & $94.37 \%$ & $93.76 \%$ & $88.81 \%$ & $80.60 \%$ & $76.35 \%$ \\
\hline es & $98.75 \%$ & $99.70 \%$ & $96.24 \%$ & $0.00 \%$ & $95.00 \%$ & $86.95 \%$ & $83.71 \%$ \\
\hline es_anco & $96.66 \%$ & $99.74 \%$ & $97.93 \%$ & $97.77 \%$ & $95.64 \%$ & $87.73 \%$ & $84.74 \%$ \\
\hline et & $93.21 \%$ & $99.07 \%$ & $89.27 \%$ & $91.13 \%$ & $74.46 \%$ & $71.24 \%$ & $59.57 \%$ \\
\hline $\mathrm{eu}$ & $100.00 \%$ & $99.99 \%$ & $94.95 \%$ & $0.00 \%$ & $83.97 \%$ & $76.87 \%$ & $70.12 \%$ \\
\hline fa & $98.74 \%$ & $99.48 \%$ & $95.75 \%$ & $95.52 \%$ & $94.33 \%$ & $83.27 \%$ & $78.84 \%$ \\
\hline fi & $89.33 \%$ & $99.77 \%$ & $94.93 \%$ & $96.07 \%$ & $88.01 \%$ & $78.73 \%$ & $74.26 \%$ \\
\hline fi_ftb & $85.02 \%$ & $100.00 \%$ & $92.96 \%$ & $0.00 \%$ & $88.60 \%$ & $78.78 \%$ & $73.34 \%$ \\
\hline $\mathrm{fr}$ & $97.73 \%$ & $99.16 \%$ & $96.27 \%$ & $0.00 \%$ & $94.87 \%$ & $87.67 \%$ & $85.03 \%$ \\
\hline fr_sequoia & $90.58 \%$ & $98.83 \%$ & $96.05 \%$ & $0.00 \%$ & $92.82 \%$ & $83.03 \%$ & $80.43 \%$ \\
\hline gl & $96.68 \%$ & $99.96 \%$ & $96.83 \%$ & $95.32 \%$ & $99.79 \%$ & $83.17 \%$ & $79.89 \%$ \\
\hline got & $26.87 \%$ & $100.00 \%$ & $94.29 \%$ & $95.23 \%$ & $80.63 \%$ & $70.46 \%$ & $62.89 \%$ \\
\hline grc & $99.34 \%$ & $100.00 \%$ & $88.35 \%$ & $77.68 \%$ & $84.01 \%$ & $68.13 \%$ & $60.82 \%$ \\
\hline grc_proiel & $42.42 \%$ & $100.00 \%$ & $96.20 \%$ & $96.54 \%$ & $86.50 \%$ & $74.26 \%$ & $68.52 \%$ \\
\hline he & $99.49 \%$ & $84.12 \%$ & $80.55 \%$ & $80.66 \%$ & $75.41 \%$ & $62.52 \%$ & $57.81 \%$ \\
\hline hi & $98.55 \%$ & $100.00 \%$ & $96.33 \%$ & $95.11 \%$ & $87.43 \%$ & $92.20 \%$ & $88.09 \%$ \\
\hline $\mathrm{hr}$ & $97.48 \%$ & $99.89 \%$ & $96.64 \%$ & $0.00 \%$ & $81.14 \%$ & $82.20 \%$ & $76.22 \%$ \\
\hline hu & $98.19 \%$ & $99.95 \%$ & $93.40 \%$ & $0.00 \%$ & $59.68 \%$ & $73.99 \%$ & $64.48 \%$ \\
\hline it & $97.02 \%$ & $99.44 \%$ & $96.92 \%$ & $96.28 \%$ & $95.44 \%$ & $87.65 \%$ & $85.36 \%$ \\
\hline it_partut & $96.62 \%$ & $99.16 \%$ & $95.16 \%$ & $94.89 \%$ & $92.60 \%$ & $82.55 \%$ & $79.02 \%$ \\
\hline ko & $90.61 \%$ & $97.91 \%$ & $91.54 \%$ & $86.50 \%$ & $97.57 \%$ & $62.80 \%$ & $55.05 \%$ \\
\hline la_ittb & $77.03 \%$ & $99.87 \%$ & $96.75 \%$ & $0.00 \%$ & $88.37 \%$ & $75.85 \%$ & $70.70 \%$ \\
\hline la_proiel & $22.81 \%$ & $100.00 \%$ & $95.62 \%$ & $95.37 \%$ & $84.94 \%$ & $68.66 \%$ & $62.19 \%$ \\
\hline lv & $94.30 \%$ & $99.66 \%$ & $91.76 \%$ & $26.01 \%$ & $69.57 \%$ & $72.57 \%$ & $64.15 \%$ \\
\hline $\mathrm{nl}$ & $93.25 \%$ & $99.66 \%$ & $94.49 \%$ & $0.00 \%$ & $89.96 \%$ & $82.82 \%$ & $77.86 \%$ \\
\hline nl_lassysmall & $81.01 \%$ & $99.81 \%$ & $96.32 \%$ & $0.00 \%$ & $93.48 \%$ & $79.35 \%$ & $74.41 \%$ \\
\hline no_bokmaal & 96. & $99.88 \%$ & $97.28 \%$ & $0.00 \%$ & $91.16 \%$ & $86.64 \%$ & $83.22 \%$ \\
\hline no_nynorsk & 93.9 & $99.94 \%$ & $96.54 \%$ & $0.00 \%$ & $91.27 \%$ & $84.78 \%$ & $81.28 \%$ \\
\hline $\mathrm{pl}$ & $99.56 \%$ & $99.17 \%$ & $95.54 \%$ & $0.00 \%$ & $77.10 \%$ & $84.51 \%$ & $79.17 \%$ \\
\hline $\mathrm{pt}$ & $90.71 \%$ & $99.53 \%$ & $96.70 \%$ & $0.00 \%$ & $92.86 \%$ & $87.45 \%$ & $84.66 \%$ \\
\hline pt_br & $96.71 \%$ & $99.82 \%$ & $97.65 \%$ & $97.57 \%$ & $99.70 \%$ & $89.23 \%$ & $87.01 \%$ \\
\hline ro & $97.47 \%$ & $99.63 \%$ & $96.58 \%$ & $9.64 \%$ & $89.17 \%$ & $86.94 \%$ & $81.59 \%$ \\
\hline $\mathrm{ru}$ & $93.09 \%$ & $99.79 \%$ & $95.48 \%$ & $94.35 \%$ & $79.82 \%$ & $82.12 \%$ & $77.38 \%$ \\
\hline ru_syntagrus & $97.29 \%$ & $99.72 \%$ & $98.10 \%$ & $0.00 \%$ & $90.25 \%$ & $88.91 \%$ & $86.14 \%$ \\
\hline sk & $76.09 \%$ & $99.93 \%$ & $94.94 \%$ & $0.87 \%$ & $69.04 \%$ & $82.74 \%$ & $77.20 \%$ \\
\hline sl & $99.59 \%$ & $99.94 \%$ & $97.38 \%$ & $14.87 \%$ & $81.19 \%$ & $87.41 \%$ & $84.31 \%$ \\
\hline SV & $96.17 \%$ & $99.88 \%$ & $95.66 \%$ & $0.00 \%$ & $89.63 \%$ & $80.57 \%$ & $76.05 \%$ \\
\hline sv_lines & $87.19 \%$ & $99.97 \%$ & $94.89 \%$ & $66.27 \%$ & $99.97 \%$ & $80.30 \%$ & $75.22 \%$ \\
\hline $\operatorname{tr}$ & $96.98 \%$ & $96.61 \%$ & $89.31 \%$ & $88.25 \%$ & $77.59 \%$ & $60.47 \%$ & $52.49 \%$ \\
\hline ur & $99.10 \%$ & $99.99 \%$ & $93.46 \%$ & $91.47 \%$ & $77.62 \%$ & $84.81 \%$ & $78.02 \%$ \\
\hline vi & $97.40 \%$ & $85.98 \%$ & $78.32 \%$ & $75.69 \%$ & $85.84 \%$ & $49.67 \%$ & $44.20 \%$ \\
\hline $\mathrm{zh}$ & $98.50 \%$ & $93.81 \%$ & $87.21 \%$ & $87.39 \%$ & $92.25 \%$ & $67.97 \%$ & $62.75 \%$ \\
\hline
\end{tabular}

Table 7: Performance of our system on development dataset. XPOS accuracy for some languages are quite low due to the format issue. 


\begin{tabular}{|c|c|c|c|c|c|c|c|}
\hline Language & Sentence & Word & UPOS & XPOS & Feats & UAS & LAS \\
\hline ar & $85.69 \%$ & $91.45 \%$ & $86.59 \%$ & $80.83 \%$ & $78.27 \%$ & $70.16 \%$ & $64.89 \%$ \\
\hline $\mathrm{bg}$ & $91.5 \%$ & $99.82 \%$ & $97.67 \%$ & $76.65 \%$ & $90.18 \%$ & $87.96 \%$ & $83.89 \%$ \\
\hline $\mathrm{ca}$ & $99.35 \%$ & $99.77 \%$ & $97.68 \%$ & $97.68 \%$ & $95.35 \%$ & $88.48 \%$ & $85.02 \%$ \\
\hline cs_cac & $99.76 \%$ & $99.94 \%$ & $98.43 \%$ & $87.99 \%$ & $83.95 \%$ & $87.54 \%$ & $83.27 \%$ \\
\hline cs_cltt & $91.99 \%$ & $99.59 \%$ & $96.65 \%$ & $83.34 \%$ & $78.42 \%$ & $80.26 \%$ & $76.08 \%$ \\
\hline cs & $95.1 \%$ & $99.99 \%$ & $98.53 \%$ & $91.45 \%$ & $87.96 \%$ & $88.14 \%$ & $84.43 \%$ \\
\hline $\mathrm{cu}$ & $37.46 \%$ & $100 \%$ & $94.8 \%$ & $95.02 \%$ & $80.78 \%$ & $73.68 \%$ & $66.91 \%$ \\
\hline da & $81.41 \%$ & $100 \%$ & $95.84 \%$ & $0 \%$ & $90.55 \%$ & $79.48 \%$ & $75.59 \%$ \\
\hline de & $78.78 \%$ & $99.61 \%$ & $92.42 \%$ & $96.74 \%$ & $78.28 \%$ & $79.17 \%$ & $74.26 \%$ \\
\hline el & $28.28 \%$ & $100 \%$ & $95.75 \%$ & $95.9 \%$ & $84.99 \%$ & $67.8 \%$ & $61.53 \%$ \\
\hline en_lines & $86.95 \%$ & $99.96 \%$ & $95.4 \%$ & $63.43 \%$ & $99.96 \%$ & $79.27 \%$ & $74.67 \%$ \\
\hline en_partut & $98.1 \%$ & $99.31 \%$ & $94.2 \%$ & $93.43 \%$ & $88.49 \%$ & $80.83 \%$ & $76.68 \%$ \\
\hline en & $78.01 \%$ & $98.98 \%$ & $94.09 \%$ & $93.46 \%$ & $92.9 \%$ & $80.8 \%$ & $77.57 \%$ \\
\hline es_ancora & $98.67 \%$ & $99.72 \%$ & $97.87 \%$ & $97.87 \%$ & $95.66 \%$ & $87.13 \%$ & $83.81 \%$ \\
\hline es & $87.08 \%$ & $99.98 \%$ & $94.88 \%$ & $62.88 \%$ & $99.98 \%$ & $80.33 \%$ & $74.89 \%$ \\
\hline et & $92.63 \%$ & $99.28 \%$ & $89.9 \%$ & $92.01 \%$ & $75.16 \%$ & $69.98 \%$ & $58.48 \%$ \\
\hline $\mathrm{eu}$ & $99.75 \%$ & $99.99 \%$ & $94.59 \%$ & $0 \%$ & $83.32 \%$ & $77.28 \%$ & $70.76 \%$ \\
\hline fa & $99.25 \%$ & $99.44 \%$ & $95.86 \%$ & $95.74 \%$ & $94.4 \%$ & $82.11 \%$ & $77.74 \%$ \\
\hline fi_ftb & $86.46 \%$ & $99.98 \%$ & $93.11 \%$ & $0 \%$ & $89.1 \%$ & $78.16 \%$ & $72.08 \%$ \\
\hline fi & $89.48 \%$ & $99.6 \%$ & $95.15 \%$ & $96.15 \%$ & $88.07 \%$ & $78.87 \%$ & $74.51 \%$ \\
\hline fr_sequoia & $82.97 \%$ & $99.19 \%$ & $96.4 \%$ & $0 \%$ & $93.33 \%$ & $82.91 \%$ & $80.3 \%$ \\
\hline fr & $92.49 \%$ & $98.84 \%$ & $95.68 \%$ & $0 \%$ & $94.27 \%$ & $83.83 \%$ & $80.38 \%$ \\
\hline gl & $96.14 \%$ & $99.98 \%$ & $96.98 \%$ & $95.82 \%$ & $99.78 \%$ & $83 \%$ & $79.79 \%$ \\
\hline got & $28.35 \%$ & $100 \%$ & $94.85 \%$ & $95.7 \%$ & $81.73 \%$ & $70.13 \%$ & $62.64 \%$ \\
\hline grc & $98.96 \%$ & $100 \%$ & $87.25 \%$ & $75.43 \%$ & $81.13 \%$ & $66.23 \%$ & $58.42 \%$ \\
\hline he & $99.49 \%$ & $80.93 \%$ & $77.34 \%$ & $77.34 \%$ & $71.84 \%$ & $58.54 \%$ & $54.25 \%$ \\
\hline hi & $99.11 \%$ & $99.99 \%$ & $96.4 \%$ & $95.8 \%$ & $87.71 \%$ & $92.31 \%$ & $88.15 \%$ \\
\hline $\mathrm{hr}$ & $95.92 \%$ & $99.88 \%$ & $96.13 \%$ & $0 \%$ & $78.71 \%$ & $82.95 \%$ & $76.63 \%$ \\
\hline hu & $94.1 \%$ & $99.75 \%$ & $92.63 \%$ & $0 \%$ & $59.13 \%$ & $74.22 \%$ & $64.37 \%$ \\
\hline id & $92.14 \%$ & $99.96 \%$ & $93.41 \%$ & $0 \%$ & $99.46 \%$ & $82.27 \%$ & $75.74 \%$ \\
\hline it & $98.76 \%$ & $99.56 \%$ & $97.29 \%$ & $97.17 \%$ & $96.02 \%$ & $88.42 \%$ & $86.01 \%$ \\
\hline $\mathrm{ja}$ & $94.64 \%$ & $93.32 \%$ & $91.04 \%$ & $0 \%$ & $93.3 \%$ & $80.71 \%$ & $79.25 \%$ \\
\hline ko & $90.99 \%$ & $98.24 \%$ & $92.51 \%$ & $88.35 \%$ & $97.9 \%$ & $68.14 \%$ & $61.14 \%$ \\
\hline la_ittb & $92.91 \%$ & $99.97 \%$ & $97.49 \%$ & $0 \%$ & $91.59 \%$ & $82.08 \%$ & $77.62 \%$ \\
\hline la_proiel & $28.28 \%$ & $100 \%$ & $95.75 \%$ & $95.9 \%$ & $84.99 \%$ & $67.8 \%$ & $61.53 \%$ \\
\hline lv & $98.8 \%$ & $99.45 \%$ & $90.09 \%$ & $26.52 \%$ & $68.6 \%$ & $69.15 \%$ & $60.94 \%$ \\
\hline nl_lassysmall & $82.8 \%$ & $99.93 \%$ & $97.74 \%$ & $0 \%$ & $95.74 \%$ & $84.92 \%$ & $81.81 \%$ \\
\hline $\mathrm{nl}$ & $76.83 \%$ & $99.79 \%$ & $92.04 \%$ & $0 \%$ & $87.45 \%$ & $77.93 \%$ & $72 \%$ \\
\hline no_bokmaal & $96.26 \%$ & $99.85 \%$ & $96.64 \%$ & $0 \%$ & $90.78 \%$ & $85.8 \%$ & $82.6 \%$ \\
\hline no_nynorsk & $80.51 \%$ & $96.99 \%$ & $28.19 \%$ & $0 \%$ & $25.88 \%$ & $23.91 \%$ & $6.57 \%$ \\
\hline $\mathrm{pl}$ & $98.73 \%$ & $98.99 \%$ & $95.66 \%$ & $0 \%$ & $77.12 \%$ & $84.05 \%$ & $78.61 \%$ \\
\hline pt_br & $96.63 \%$ & $99.83 \%$ & $97.42 \%$ & $97.42 \%$ & $99.7 \%$ & $87.57 \%$ & $85.41 \%$ \\
\hline $\mathrm{pt}$ & $91.67 \%$ & $99.34 \%$ & $96.51 \%$ & $0 \%$ & $92.24 \%$ & $85.15 \%$ & $82.03 \%$ \\
\hline ro & $94.79 \%$ & $99.64 \%$ & $96.71 \%$ & $9.43 \%$ & $89.06 \%$ & $86.68 \%$ & $81.19 \%$ \\
\hline ru_syntagrus & $97.97 \%$ & $99.69 \%$ & $98.2 \%$ & $0 \%$ & $90.38 \%$ & $89.36 \%$ & $86.83 \%$ \\
\hline $\mathrm{ru}$ & $95.75 \%$ & $99.81 \%$ & $95.49 \%$ & $95.24 \%$ & $80.24 \%$ & $81.58 \%$ & $76.53 \%$ \\
\hline sk & $82.04 \%$ & $99.98 \%$ & $94.22 \%$ & $0.9 \%$ & $68.49 \%$ & $81.58 \%$ & $76.23 \%$ \\
\hline sl & $99.24 \%$ & $99.93 \%$ & $96.92 \%$ & $14.55 \%$ & $81.73 \%$ & $85.74 \%$ & $82.19 \%$ \\
\hline Sv_lines & $87.08 \%$ & $99.98 \%$ & $94.88 \%$ & $62.88 \%$ & $99.98 \%$ & $80.33 \%$ & $74.89 \%$ \\
\hline SV & $94.92 \%$ & $99.84 \%$ & $96.05 \%$ & $0 \%$ & $89.81 \%$ & $82.31 \%$ & $77.7 \%$ \\
\hline $\operatorname{tr}$ & $96.32 \%$ & $97.13 \%$ & $91.3 \%$ & $90.45 \%$ & $78.62 \%$ & $61.69 \%$ & $53.08 \%$ \\
\hline ur & $97.67 \%$ & $100 \%$ & $93.17 \%$ & $91.23 \%$ & $78.21 \%$ & $85.22 \%$ & $78.61 \%$ \\
\hline vi & $92.44 \%$ & $83.8 \%$ & $75.84 \%$ & $72.94 \%$ & $83.56 \%$ & $46.16 \%$ & $40.89 \%$ \\
\hline $\mathrm{zh}$ & $98.5 \%$ & $94.57 \%$ & $88.36 \%$ & $88.4 \%$ & $92.9 \%$ & $70.35 \%$ & 65.15 \\
\hline
\end{tabular}

Table 8: Official performance of our system on big treebanks. For language no_nynorsk, we used the model trained on another language, thus got very poor result. 


\begin{tabular}{|c|c|c|c|c|c|c|c|}
\hline Language & Sentence & Word & UPOS & XPOS & Feats & UAS & LAS \\
\hline fr_partut & $98.5 \%$ & $98.88 \%$ & $95.26 \%$ & $95.04 \%$ & $90.07 \%$ & $84.2 \%$ & $80.06 \%$ \\
\hline ga & $94.75 \%$ & $99.64 \%$ & $90.79 \%$ & $89.9 \%$ & $71.95 \%$ & $76.51 \%$ & $66.49 \%$ \\
\hline gl_treegal & $86.74 \%$ & $97.91 \%$ & $91.89 \%$ & $86.48 \%$ & $83.83 \%$ & $73.44 \%$ & $67.97 \%$ \\
\hline kk & $71.84 \%$ & $94.45 \%$ & $56.76 \%$ & $57.13 \%$ & $36.46 \%$ & $44.67 \%$ & $23.99 \%$ \\
\hline la & $98.14 \%$ & $99.99 \%$ & $88.57 \%$ & $68.96 \%$ & $67.45 \%$ & $59.83 \%$ & $48.33 \%$ \\
\hline s__sst & $17.58 \%$ & $100 \%$ & $91.78 \%$ & $13.97 \%$ & $75.6 \%$ & $56.73 \%$ & $49.53 \%$ \\
\hline ug & $67.13 \%$ & $96.94 \%$ & $74.76 \%$ & $76.87 \%$ & $96.94 \%$ & $54.54 \%$ & $34.57 \% \%$ \\
\hline uk & $92.49 \%$ & $99.82 \%$ & $90.08 \%$ & $8.95 \%$ & $62.26 \%$ & $73.52 \%$ & $65.17 \%$ \\
\hline ar_pud & $99.4 \%$ & $89.08 \%$ & $69.85 \%$ & $0 \%$ & $21.16 \%$ & $53.74 \%$ & $44.31 \%$ \\
\hline cs_pud & $96.13 \%$ & $98.48 \%$ & $96.32 \%$ & $87.99 \%$ & $83.56 \%$ & $85.12 \%$ & $79.76 \%$ \\
\hline de_pud & $88.4 \%$ & $96.28 \%$ & $83.82 \%$ & $20.29 \%$ & $30.75 \%$ & $75.61 \%$ & $69.19 \%$ \\
\hline en_pud & $98.06 \%$ & $99.59 \%$ & $94.46 \%$ & $93.43 \%$ & $91.38 \%$ & $83.56 \%$ & $79.88 \%$ \\
\hline es_pud & $94.88 \%$ & $99.24 \%$ & $88.01 \%$ & $0 \%$ & $54.03 \%$ & $84.27 \%$ & $77.09 \%$ \\
\hline fr_pud & $96.55 \%$ & $96.63 \%$ & $87.56 \%$ & $0 \%$ & $57.52 \%$ & $78.74 \%$ & $73.67 \%$ \\
\hline hi_pud & $1.17 \%$ & $92.38 \%$ & $79.04 \%$ & $34.5 \%$ & $13.38 \%$ & $54.7 \%$ & $43.46 \%$ \\
\hline it_pud & $93.97 \%$ & $99.08 \%$ & $93.19 \%$ & $2.48 \%$ & $57.18 \%$ & $87.72 \%$ & $84.41 \%$ \\
\hline ja_pud & $96.6 \%$ & $93.57 \%$ & $91.7 \%$ & $0 \%$ & $54.72 \%$ & $82.04 \%$ & $81.25 \%$ \\
\hline pt_pud & $97.32 \%$ & $98.51 \%$ & $88 \%$ & $0 \%$ & $58.8 \%$ & $78.75 \%$ & $72.85 \%$ \\
\hline ru_pud & $97.23 \%$ & $97.15 \%$ & $85.67 \%$ & $79.23 \%$ & $37.33 \%$ & $77.5 \%$ & $69.46 \%$ \\
\hline sv_pud & $94.11 \%$ & $98.39 \%$ & $92.54 \%$ & $0 \%$ & $69.4 \%$ & $75.94 \%$ & $70.76 \%$ \\
\hline tr_pud & $93 \%$ & $94.94 \%$ & $68.8 \%$ & $0 \%$ & $22.41 \%$ & $52.75 \%$ & $31.53 \%$ \\
\hline bxr & $90.62 \%$ & $97.46 \%$ & $49.11 \%$ & $0 \%$ & $39.9 \%$ & $36.42 \%$ & $17.08 \%$ \\
\hline hsb & $72.93 \%$ & $94.32 \%$ & $63.89 \%$ & $0 \%$ & $35.05 \%$ & $37.58 \%$ & $24.58 \%$ \\
\hline kmr & $92.91 \%$ & $91.45 \%$ & $58.03 \%$ & $56.45 \%$ & $31.56 \%$ & $33.98 \%$ & $25.85 \%$ \\
\hline sme & $98.09 \%$ & $96.52 \%$ & $53.98 \%$ & $57.7 \%$ & $30.7 \%$ & $31.22 \%$ & $17.1 \%$ \\
\hline
\end{tabular}

Table 9: Official performance of our system on small treebanks, PUD treebanks and suprise languages.

\subsection{Official Results and Analysis}

Detailed numbers for official runs on the test set (Nivre et al., 2017) are listed in Table 8 and Table 9 .

Our system ranked the $15^{\text {th }}$ among the 33 submissions. Unfortunately, we found that for one language (no_nynorsk), we used the model trained on another language, therefore the performance is poor. Changing to the correct model would change our results from $67.87 \%$ averaged macro F1 score to $68.78 \%$. For two languages la and grc_proiel, we trained the 1-endpoint-crossing parser, but used the projective parser for testing due to memory issue. On the development dataset, we found that such strategy lost about $0.5 \%$ LAS due to the inconsistent decoding algorithms between training and testing. For PUD treebanks that have no corresponding training portion, we used the model trained on the non-PUD dataset, e.g., used the model trained on en to parse en pud.

Regarding speed, our parser is not optimized for running time nor memory. It spent 67 hours to parse all the languages using 10 threads. The peak memory usage is about $89 \mathrm{~GB}$ when parsing grc proiel. The most time consuming part in our system is feature generation that has a complexity of $O\left(n^{3} T\right)$, where $T=260$ is the number of templates.

\section{Conclusion and Future Work}

We described our system for the universal dependency parsing task that relies heavily on feature engineering for each component in the pipeline. Our system achieves reasonable performance. An important observation we have is regarding the pretrained word embeddings. Unlike neural net based parsers that can effectively use large unlabeled data by pretrained word embedding, pictures of semi-supervised learning approaches for feature engineering based systems are unclear. Though we tried different ways in our work, the improvement is quite limited. In our future work, we plan to combine our system with neural net based approaches and explore some other semi-superivsed learning techniques.

\section{References}

Liang Huang and David Chiang. 2005. Better k-best parsing. In Proceedings of the Ninth International Workshop on Parsing Technology. Association for Computational Linguistics, Stroudsburg, PA, USA, Parsing '05, pages 53-64. http://dl.acm.org/citation.cfm?id=1654494.1654500.

Terry Koo and Michael Collins. 2010. Efficient thirdorder dependency parsers. In Proceedings of the 48th Annual Meeting of the Association for Computational Linguistics. Association for Computa- 
tional Linguistics, Uppsala, Sweden, pages 1-11. http://www.aclweb.org/anthology/P10-1001.

John D. Lafferty, Andrew McCallum, and Fernando C. N. Pereira. 2001. Conditional random fields: Probabilistic models for segmenting and labeling sequence data. In Proceedings of the Eighteenth International Conference on Machine Learning. Morgan Kaufmann Publishers Inc., San Francisco, CA, USA, ICML '01, pages 282-289. http://dl.acm.org/citation.cfm?id=645530.655813.

Guillaume Lample, Miguel Ballesteros, Sandeep Subramanian, Kazuya Kawakami, and Chris Dyer. 2016. Neural architectures for named entity recognition. CoRR abs/1603.01360. http://arxiv.org/abs/1603.01360.

Ryan McDonald, Koby Crammer, and Fernando Pereira. 2005. Online large-margin training of dependency parsers. In Proceedings of the 43rd Annual Meeting of the Association for Computational Linguistics (ACL'05). Association for Computational Linguistics, Ann Arbor, Michigan, pages 91-98. https://doi.org/10.3115/1219840.1219852.

Joakim Nivre et al. 2017. Universal Dependencies 2.0 CoNLL 2017 shared task development and test data. LINDAT/CLARIN digital library at the Institute of Formal and Applied Linguistics, Charles University, Prague, http://hdl.handle.net/ 11234/1-2184. http://hdl.handle.net/11234/12184.

Emily Pitler. 2014. A crossing-sensitive third-order factorization for dependency parsing. Transactions of the Association for Computational Linguistics 2:41-54. https://www.transacl.org/ojs/index.php/tacl/article/view/193.

Emily Pitler, Sampath Kannan, and Mitchell Marcus. 2013. Finding optimal 1-endpointcrossing trees. Transactions of the Association for Computational Linguistics 1:13-24. https://www.transacl.org/ojs/index.php/tacl/article/view/23.

Daniel Zeman, Filip Ginter, Jan Hajič, Joakim Nivre, Martin Popel, Milan Straka, and et al. 2017. CoNLL 2017 Shared Task: Multilingual Parsing from Raw Text to Universal Dependencies. In Proceedings of the CoNLL 2017 Shared Task: Multilingual Parsing from Raw Text to Universal Dependencies. Association for Computational Linguistics, pages 1-20. 